\title{
Milan Rastislav Štefánik, personnage central de l'histoire slovaque
}

\section{Michal Kšiňan}

\section{(2) OpenEdition \\ 1 Journals}

Édition électronique

URL : http://journals.openedition.org/rbnu/716

DOI : $10.4000 /$ rbnu. 716

ISSN : 2679-6104

Éditeur

Bibliothèque nationale et universitaire de Strasbourg

\section{Édition imprimée}

Date de publication : 1 novembre 2017

Pagination : 104-107

ISBN : 9782859230678

ISSN : 2109-2761

\section{Référence électronique}

Michal Kšiňan, « Milan Rastislav Štefánik, personnage central de l'histoire slovaque », La Revue de la BNU [En ligne], 16 | 2017, mis en ligne le 01 juillet 2019, consulté le 11 décembre 2020. URL : http:// journals.openedition.org/rbnu/716; DOI : https://doi.org/10.4000/rbnu.716

\section{(c) (i) (2)}

La Revue de la BNU est mise à disposition selon les termes de la Licence Creative Commons Attribution - Pas d'Utilisation Commerciale - Partage dans les Mêmes Conditions 4.0 International. 
ASTRONOMIE PHYSIQLe. - Observation de léclipse totale de Soleil à l'île Vavau (Archipel Tonga), le 28 avril I $9 \mathrm{r}$ r. Note (') de M. Milax Š́terixik, présentée par M. Henri Poincaré.

La zone de la totalité de cette éclipse, partant d'Australie à $8^{\mathrm{h}} 5 \mathrm{o}^{\mathrm{m}} \mathrm{T}$. $\mathrm{m}$. P. aux environs nord-est de Melbourne se dirigeait vers l'île Norfolk, l'archipel Tonga, les îles Danger, les Samoa américaines, pour se perdre enfin près de la côte du Mexique vers $12^{\mathrm{h}}{ }^{1} 7^{\mathrm{m}}$. L'ile Vavau du groupe du même nom offrait des avantages appréciables par la proximité de la ligne centrale de l'éclipse et ses communications régulières avec le monde extérieur.

Pour y parvenir en temps opportun, j'ai dù quitter le 24 février Tahiti oú je poursuivais depuis presque un an des études astronomiques et passer par l'archipel de Cook, la Nouvelle-Zélande et Tongatabu.

A la date de mon arrivée, le 7 avril, la saison des pluies venait de prendre fin, la chaleur se modérait, ce qui facilita les travaux préparatoires. Mes instruments étaient installés sur une colline peu élevée $\left(5 \mathrm{o}^{\mathrm{m}}\right.$ environ) et sitnée à quelques centaines de mètres du port Neiafu (Lat. S. $18^{\circ} 39^{\prime} 17^{\prime \prime}, 5$; Long. W. $\left.173^{\circ} 58^{\prime} 55^{\prime \prime}\right)$.

Soucieux de ne pas toucher à mon installation astronomique au mont Faiere à Tahiti, je n'ai enlevé de cette station que les pièces optiques de quelques instruments et, grâce au bienveillant concours du commandant et des officiers de la canonnière Zelée, j'ai construit l'appareil ci-dessous indiqué. Un tube octogonal en bois portait à une extrémité un objectif photographique Schaer $\left(27^{\mathrm{cm}}\right.$ d'ouverture utile et $2^{\mathrm{m}}, 35$ de distance focale $)$ et à l'autre extrémité une chambre photographique. Sur le milieu du tube on ajusta un solide collier en fer présentant deux tourillons et supportés par une fourche faite de deux cornières en fer. La fourche reposait sur deux piliers maçonnés avec du corail pris sur place et du ciment dont j'avais eu la précaution de me munir en Nouvelle-Zélande. Dans un pilier, je scellai un appareil réglable en azimut et en hauteur, sur la face supérieure inclinée de l'autre pilier j'ai scellé une plaque métallique, sur laquelle s'appuyait normalement le tourillon inférieur de la fourche. Auprès de ce tourillon se trouvait calé, perpendiculairement à l'axe de la fourche, une demi-roue de $\mathrm{I}^{\mathrm{m}}$, 5 o de diamètre. Elle était reliée par une lame d'acier avec le mouvement

(1) Présentée dans la séance du 12 février 1912.

C. R., 1912,1 * Semestre. (T. 154, N•9.) 73 


\section{$\overline{\text { SLOVAQUIE }}$ \\ MILAN RASTISLAV ŠTEFÁNIK, PERSONNAGE CENTRAL DE L'HISTOIRE SLOVAQUE \\ PAR MICHAL KŠIŇAN}

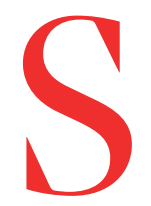

en Slovaquie ou Haute-Hongrie en 1880, il s'installe à Paris à l'âge de 24 ans et prend la nationalité française en 1912. Pourtant son souvenir n'est présent dans sa « seconde patrie " qu'à travers quelques lieux de mémoire mineurs, comme une petite place portant son nom dans le $16^{\mathrm{e}}$ arrondissement de Paris, ou encore sa statue dans les jardins de l'Observatoire de Meudon. Dans le monde ou en Europe, seuls les cercles restreints qui sont familiers avec les régions d'Europe centrale connaissent sa carrière diplomatique, politique et militaire.

Avant le premier conflit mondial toutefois, ses succès dans le domaine de l'astronomie lui valent une certaine notoriété dans la communauté scientifique internationale. Štefánik publie, seul ou comme coauteur, onze articles dans des revues savantes de haute renommée, notamment les Comptes rendus de l'Académie des sciences. La précarité financière et des problèmes de santé l'empêchent de pousser plus loin ses recherches. Il se met peu à peu à défendre les intérêts français en Équateur et dans l'Océanie française, ce qui lui ouvre les portes du Quai d'Orsay. Pour ses mérites, il se voit distingué par la croix de chevalier de la légion d'honneur en 1914.

Štefánik n'est pas un homme de science ennuyeux. Ce causeur incomparable profite de ses nombreuses expériences vécues pour alimenter des conversations qui font rire ses interlocuteurs. Voici par exemple une de ses anecdotes : «Il [Štefánik] fut chargé d'observer une éclipse de soleil qui devait être complètement observable en Polynésie. Il n'hésita pas et partit pour une petite île perdue en (sic) archipel polynésien. Débarqué avec son télescope, il annonça au chef indigène que le soleil allait disparaître, mais le gouvernement (sic) de la République (conscient de ses devoirs de protecteur) l'envoyait lui, Štefánik, pour faire réapparaître la lumière solaire. Tout se passa comme prévu. Stupéfait de la nuit tombée en plein Midi, plus suffoqué encore par le retour de l'astre du jour dû au magicien, le chef se prosterna probablement devant l'être surhumain détenteur des sortilèges. Mais 
par humilité, Štefánik n'avait jamais voulu avouer ce geste du sauvage émerveillé » ${ }^{1}$. S'il a le faible d'exagérer parfois pour se rendre intéressant et parader un peu, tout le monde sait que ses histoires sont inspirées par la très grande richesse de son parcours professionnel et personnel. Les souvenirs qu'on a pu garder de lui soulignent le caractère romanesque et original de sa vie. Par son influence personnelle, il se gagne alors des sympathies dans les plus hautes sociétés italienne et française. C'est à cette époque un ornement des salons.

Son ascension politique s'accélère au cours de la Première Guerre mondiale, lorsqu'il prend la tête du mouvement nationaliste tchécoslovaque aux côtés d'Edouard Beneš et de Thomas Masaryk, avec pour objectif de démembrer l'Autriche-Hongrie afin de créer la nouvelle Europe, et surtout une Tchécoslovaquie indépendante. Štefánik profite de ses acquis de l'époque d'avant-guerre : ses réseaux parmi les diplomates lui sont très utiles. Il en est de même pour sa capacité à convaincre et gagner ses interlocuteurs qui deviennent ensuite des partisans de la cause tchécoslovaque et l'introduisent auprès d'hommes politiques de premier rang. Il ajoute de nouveaux éléments à son prestige : fasciné par les nouvelles inventions, il s'engage dans l'aviation et lutte sur les fronts occidental et serbe. Les pilotes jouissent à l'époque d'un statut de héros. De plus, Štefánik devient en trois ans et demi général de brigade ; sa carrière militaire suscite aujourd'hui encore l'admiration.

Sa principale préoccupation pendant la guerre est la mise sur pied d'une armée tchécoslovaque qui lutterait aux côtés de l'Entente pour montrer la volonté de son peuple d'aller vers l'indépendance. Les prisonniers de guerre d'origines tchèque et slovaque de l'armée austro-hongroise représentent le principal réservoir pour ce recrutement. La double monarchie engageait ses soldats avant tout sur les fronts italien et russe, d'où la nécessité de missions de Štefánik dans l'empire tsariste et le pays transalpin. Bien qu'on ait voulu créer au départ l'armée tchécoslovaque en France, étant donné l'importance du front occidental, elle naît finalement dans trois États de l'Entente : la France, l'Italie et la Russie. Štefánik, le «ministre pour les situations extraordinaires » ou encore « le pompier qu'on envoie éteindre différents foyers d'incendies », est le plus actif du triumvirat tchécoslovaque, et il intervient dans de nombreux pays alliés aux quatre coins du monde afin de régler divers problèmes qui surgissent concernant cette armée. On le voit en Serbie (1915), Italie (1916), Roumanie (1916), Russie (1916/1917), aux États-Unis (1917), de nouveau en Italie (1918 et 1919), en Russie en passant par les États-Unis et le Japon (1918/1919). La valeur propagandiste de l'armée tchécoslovaque dépasse largement sa contribution militaire à la victoire finale en 1918. Les leaders de l'Entente sont convaincus que la création de l'armée tchécoslovaque affaiblit la machine militaire austro-hongroise et amplifie les tendances révolutionnaires des peuples opprimés de la maison des Habsbourg. Pour cette raison, à la fin de la guerre, les Alliés récompensent les Tchèques et les Slovaques qui obtiennent leur indépendance. Personne ne met en question l'apport de Štefánik à la naissance du nouvel État, dont il devient le premier ministre de la Guerre.

Štefánik trouve la mort à l'âge de 39 ans, en 1919, peu après la cessation des hostilités, lors de son retour dans sa patrie "libérée ", dans un accident d'avion. Sa fin romanesque et entourée de théories du complot ne fait que renforcer sa popularité. La nouvelle République ne se fait pas faute de mettre en place un culte important voué à son "père fondateur" décédé prématurément. Sous le régime communiste, le nom de Štefánik se retrouve sur la liste noire à cause de son engagement anti-bolchevique à la tête de l'armée tchécoslovaque dans la guerre civile russe. Les idéologues communistes décrivent la naissance de la Tchécoslovaquie en 1918 comme le résultat de la révolution d'octobre 1917, voulant ainsi détruire la légende " bourgeoise ", y compris celle de Štefánik. Tout cela augmente encore sa popularité après 1989. Il se voit paré de l'auréole de la personnalité contestée. Aujourd'hui, Štefánik jouit du statut d'un héros - voire $d u$ héros national slovaque.

\section{NOTE}

1- Archives du ministère des Affaires étrangères, Paris-Courneuve, PA-AP 21, Robert de Billy, vol. 5, Le général Štefánik, les souvenirs de Robert de Billy, diplomate français. 
566 ACADEME DES SCIExCES.

d'horlogerie placé sur un support voisin. Sur l'axe d'une des roves qui dépassait la paroi de la bolte d'horlogerie, on avait emmanché solidement une longue vis sur laquelle se déplaçait un écrou relié à la lame de transmissiou par un étrier en acier. La rigiditè de '’utrier et de la lame empéchai l'écrou de tourner avec la vis et l'obligeait à se déplacer longitudimalement, lorsque la vis tournait. Le fonctionnement de cet appareil improvisé a montré une stabilité et une règularité tout à fait satisfaisantes.

Sur le corps de la lunette j’adaptai un spectrographe à riseat. Un réfracto-réflecteur Schaer de 6 pouces muni d'un prisme objectif Schaer fut installẻ sur un trépied.

Bclipse. - Le jour de l'éclipse le ciel était d'abord couvert, mais bientôt le vent commencait à balayer les nuages.

D'aprés les calculs, le premier eontact devait avoir lieu à Vavau (t. L.)

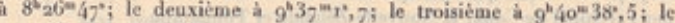
quatrième à $10^{\text {h }} 58^{*}=48^{*}$.

Je nèglige de décrire les phénomènes intéressants mais habituels qui se produisent pendant que l'éclipse n'est que partielle. Je ne veux mentionner que le magnifique halo, les aigrettes entrevues, sinsi que le jeu féerique des nuages irisés, au travers desquels j'admirai le eroissant solaire qui diminuait peu à peu pour disparaltre derrière un écran de nuages à l'approche du deuxième contact.

Vers $9^{\mathrm{k}} 36^{\mathrm{m}}$ jot environ, une obscurité enveloppa subitement le ciel et la terre et produisit l'impression, chez tous les obsersateurs, que la totalité a commencé; mais, 15 secondes plus tard, pendant un isstant, le mince croissant du soleil non éclipsé réapparut. La totalité ne devait done commencer que 5 ou 6 secondes avant l'heure caleulée. L'inondation subite de la lumière nous fit pressentir le troisième contact. Si cette impression est juste, la durće de l'éelipse ne correspondrait pas non ples au temps prévu; elle était de quelques secondes plus courte.

Travaux pendant l'éclipse. - Mon intention était d'obtenir progressivement une série de photographies du soleil éclipsé en utilisant différents éerans et des plaques sensibilisées. Vu l'état du ciel, j'ai modifie mon programme au dernier moment et, profitant des éclaircies pendant la totalité, je me suis contenté d'obtenir, par les procédés habituels, les photographies de la couronne solaire. L'une, de courte durée de pose, me donne la chromosphère et la couronne intérieure; d'autres surtout la couronne extérieure. Sur la photographie(notamment prise au commencement
SËANCK DU 26 FÉVRIER 1912.

de la totalité), on remarque que dans la région qui entoure le pôle Nord, il $y$ a à peine de trace de la matière coronale, tandis que celle da Sud est sillonnée de nombreux rayons lumineux qui s'infléehissent (peut-être sous l'influence du champ magnétique du soleil) vers Est et Onest. Ces fléchissements sont d'autant plus prononcés qu'on s'éloigne du pôle pour aboutir en quatre grands jets lumineux et symétriques des deux côtés de l'équateur, donnant a la couronne la forme habituelle et earactéristique des époquer de minima des taches. Plusieurs des rayons se détachent nettement sur le fond lumineux de la couronne intérieure, et on peut les suivre parfois jusqu' 'à la chromosphère elle-mêne. Leurs longueurs maxima nettement visibles sur mes photographies atteignent $30^{\circ}$, mais on les soupconne bien plus loin. Des traces de perturbation se manifestent aux environs Sud-Est et Sud-Ouest, oú l'on aperçoit en mème temps des protubérances, encadrées souvent par des jets remarquables.

Etudes spectroscopiques. - L'étude de la couche renversante n'était pas possible, le ciel étant voilé au moment précis des deuxième et troisième contacts. Par contre, j'ai obtenu des photographies du spectre de la coutronne avec le spectrographe, qui était réglé de manière à enregistrer une partie restreinte de la région oú se trouvent les raies de 5303 de longueur d'onde.

La photographie obtenue montre un spectre continu, sur le fond daquel se détache la raie indiquíe. En examinant attentivement cette raie (qui est du reste faible, mais large ), on est tenté de lui trouver plutat l'aspect d'une bande dégradée que d'une simple raie.

Pendant quelques instants je pus jeter un coup d'ceil dans mon 6 pouces muni d'un prisme objectif. Mon attention fut attiréc immédiatement par l'image monochromatique de la couronne extérieure formée par le coronium, tandis que je la cherchais vainement dans les autres radiations. La distribution de la matière eoronale antour du disque solaire est, d'après ces observations visuelles, analogue à celle que nous montrent les photographies; cependant, il m'a semblé apercevoir la prisence de coronium dans les régions éloignées assez uniformément répandue autour du disque, tandis qu'en s'en approchant il disparaissait aux poles d'accord avec les photographies.

Jai, en terminant, un devoir agréable à remplir, e'est d'adresser mes remerciments, d'abord au Bureau des Longitudes, dont la confianee m'a honoré et m'a facilité mon voyage, et aussi à touss ceux qui m'ont secondé dans mon entreprice. 